\title{
Transient effects of jumping lunge on sand on balance ability in healthy adults: a preliminary study
}

\author{
Min-hyeok Choi ${ }^{a, \dagger}$, Ho-jin Shin ${ }^{a, \dagger}$, Suk-Chan Hahm ${ }^{b}$, Min-Goo Lee ${ }^{c_{,}}$, Hwi-young Cho ${ }^{d_{, \star}}$ \\ ${ }^{a}$ Department of Health Science, Gachon University Graduate School, Incheon, Republic of Korea \\ ${ }^{b}$ Graduate School of Integrative Medicine, CHA University, Seongnam, Republic of Korea \\ ${ }^{c}$ Department of Physiology, Korea University College of Medicine, Seoul, Republic of Korea \\ dDepartment of Physical Therapy, College of Health Science, Gachon University, Incheon, Republic of Korea
}

Objective: The purpose of this study was to show the temporary effects of performing jumping lunges on a sand surface on static balance and dynamic balance.

Design: Randomized controlled trial.

Methods: Twenty healthy subjects volunteered in the study and was randomly assigned into either the sand group $(\mathrm{n}=10)$ or the control group $(n=10)$. The subjects in the sand group performed jumping lunges on a sand surface and the subjects in the control group performed jumping lunges on a firm surface. The intervention was performed for 3 sets of 8 repetitions by both groups. To measure static balance, the force plate was employed to measure the center of pressure $(\mathrm{CoP})$ area, and the CoP velocity during one-legged standing. Anterior, postero-medial, postero-lateral movements was assessed using the Star Excursion Balance Test (SEBT) to measure dynamic balance.

Results: After the intervention, the sand group showed statistically significant improvements on all variables (CoP area, CoP velocity) in static balance $(p<0.05)$. There were statistically significant changes in CoP area and CoP velocities between the two groups $(p<0.05)$. In the sand group, there were significant improvements in the postero-medial and postero-lateral directions $(p<0.05)$ except for anterior direction on dynamic balance. In the control group, there was a significant improvement in the postero-lateral and anterior directions $(p<0.05)$. In comparison of the two groups, there was no statistically significant improvement in all variables.

Conclusions: This study demonstrated that performing jumping lunges on a sand surface was effective in improving static and dynamic balance temporarily.

Key Words: Plyometric exercise, Postural balance, Young adult

\section{Introduction}

Balance is an ability to maintain the center of mass within the base of support. Loss of balance increases the chances of falling and limits mobility [1]. Thus, maintaining and im- proving balance ability is a very important in performing daily life activities, such as walking, running, and climbing stairs. To improve balance ability, many interventions such as nerve mobilization, taping, resistance training, tai-chi, pilates, and plyometric exercises have been investigated [2,3].

Received: 13 November, 2018 Revised: 12 December, 2018 Accepted: 13 December, 2018

Corresponding author: Min-Goo Lee (ORCID https://orcid.org/0000-0003-2822-4973)

Department of Physiology, Korea University College of Medicine, 73 Inchon-ro, Seongbuk-gu, Seoul 02841, Republic of Korea

Tel: 82-2-2286-1194 Fax: 82-2-925-5492 E-mail: mingoolee@korea.ac.kr

Corresponding author: Hwi-young Cho (ORCID https://orcid.org/0000-0003-0465-9665)

Department of Physical Therapy, College of Health Science, Gachon University, 191 Hambangmoe-ro, Yeonsu-gu, Incheon 21936, Republic of Korea Tel: 82-32-820-4560 Fax: 82-32-820-4420 E-mail: hwiyoung@gachon.ac.kr

*These authors contributed equally to this work as corresponding authors

${ }^{+}$These two authors contributed equally to this work as co-first author.

(c) This is an Open-Access article distributed under the terms of the Creative Commons Attribution Non-Commercial License (http://creativecommons.org/licenses/ by-nc/4.0) which permits unrestricted non-commercial use, distribution, and reproduction in any medium, provided the original work is properly cited.

Copyright $\odot 2018$ Korean Academy of Physical Therapy Rehabilitation Science 
Among those interventions, plyometric exercises have been commonly used for athletes to improve their balance, which is important for making good results on the field [4,5]. Also, performing exercises on different surfaces is a common method used to improve balance [6].

It has been reported that plyometric exercises play a significant role in improving balance ability $[5,6]$. However, most of previous studies were focused the long-term effects, thereby the short-term effects or immediate effects following plyometric intervention was uncertain until the present [7]. Moreover, individual plyometric programs have not been studied extensively and therefore, the scientific evidence of the effects and benefits are insufficient.

Balance exercise performance on an unstable surface is more effective in improving balance than using common firm surfaces. However, most of studies used tools such as Togu only, and other studies used various surfaces, such as firm surfaces, grass, and artificial grass, and were compared to each other only $[8,9]$. Sand, which is one of the most common surfaces among unstable surfaces, is known to be a useful surface for deriving many good results after exercise on persons with articular problems, ordinary people, and elite athletes due to its characteristics, such as small particles and its ability to spreading onto the surface [5].

There are a few studies about the effect of performing plyometric exercises on a sand surface, but since the studies selected elite athletes, such as beach soccer players as subjects, it is difficult to apply the results on ordinary people or patients [5,10]. Previous studies mostly included athletes as subjects and did not focus on balance ability on sand surface, although its characteristics are useful for ordinary people and patients since the surface derives less fatigue on the musculoskeletal system [5,6,8-10]. It is important to prove that balance ability can be improved by exercising on a sand surface, which is easily accessible, in order to develop a good balance program for the general population and patients.

Thus, the purpose of this study was to examine the temporary effect of performing plyometric exercises on a sand surface on balance ability and to compare the effect of exercising on a sand and firm surface.

\section{Methods}

\section{Experimental design}

This study was designed as a single blinded, randomized control trial. The subjects were assigned into two groups randomly using Random Allocation Software. One physical therapist performed the intervention and other physical therapist conducted the measurement under a blinded condition.

\section{Subjects}

Thirty-five people who are living in Korea volunteered in the study and 20 people ( 15 male and 5 female) who satisfied the criteria were randomly selected as subjects using simple random sampling. The inclusion criteria were subjects who were older than 18 years old, did not experience plyometric exercise before, and those interested plyometric exercises were selected. The exclusion criteria were 1) those with orthopaedic or neurologic diseases and 2) those with visual or vestibular deficits that may affect balance test performance, 3) person having lower extremity injury within at least six months of the study, 4) person having history of osteomyoarticular lesions or previous fracture or surgery to the lower extremity $[11,12]$. Prior to participating in the study, subjects were informed about the purpose and procedure of the study and then signed the consent forms. The study was approved by Institutional Review Board of Gachon University (IRB No. 1044396-201805-HR-119-01).

\section{Procedures}

The subjects were randomly assigned into one of two groups, either the sand group or the control group. A week before participating in the experiment, the subjects learned and were educated about the jumping lunge. Static and dynamic balances were measured using a force plate and the Star Excursion Balance test (SEBT). The subjects rested for 5 minutes after the pre-measurement session. To perform the intervention, the subjects stretched for warm-up and performed jumping lunges on the sand or firm surface. The subjects rested for 1 minute after a jumping lunge and then were measured just as the pre-measurement process. The measuring process took approximately 45 minutes.

\section{Intervention}

The subjects placed their non-dominant leg behind till the knee was right below their body when they performed the lunge and with their hands on their waist. The subjects stood their upper body straight and gazed their front side. When the subjects performed the lunge, they bent their knees until $90^{\circ}$ and then jumped (Figure 1A). They switched their legs during the jump (Figure 1B). The evaluator who did not participate in the measurement supervised the subjects per- 


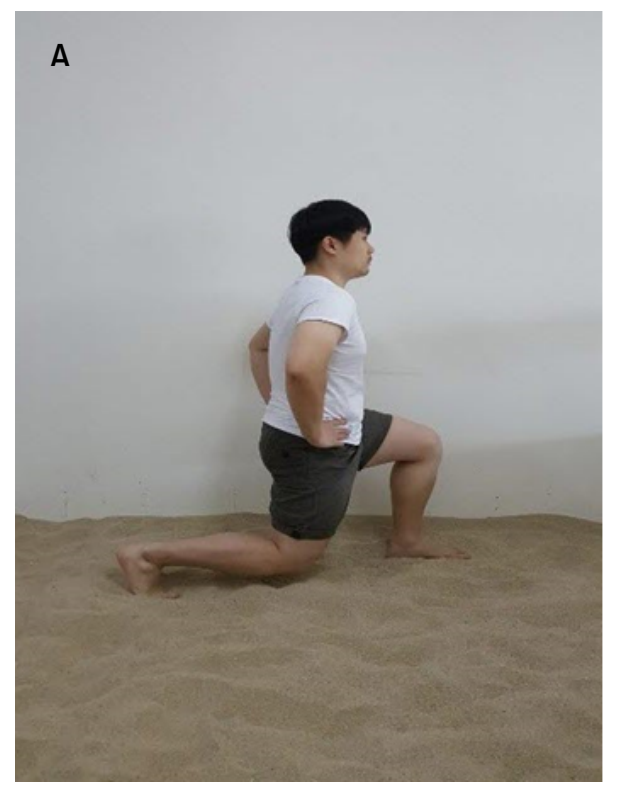

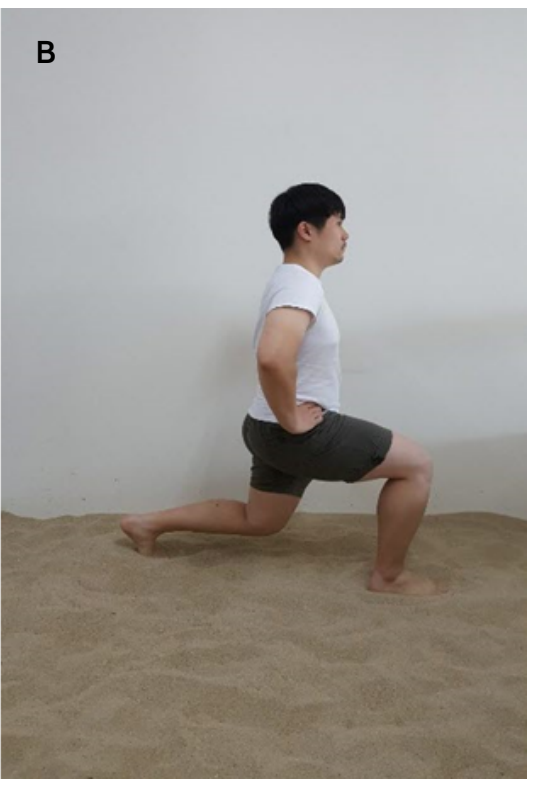

Figure 1. (A) Jumping lunge in action. (B) Jumping lunge in end position. The subjects bent their knees till $90^{\circ}$ and then jumped. The subjects switched their legs during jumping and landed. forming the jumping lunge. If they failed to maintain balance it was counted as one, therefore, the subjects were required to maintain their balance as best as possible, and they performed the lunges with their preferred speeds. The jumping lunge was done for 3 sets of 8 repetitions total, and a one-minute break was provided between the sets.

\section{Outcome measurement}

\section{Static balance measurements}

To measure static balance, the center of pressure (CoP) was measured using a force plate (FDM-SX Pressure Plate; NORAXON, Scottsdale, AZ, USA) and the signal was processed using MyoResearch 3 software (Noraxon Inc., Scottsdale, AZ, USA). The measured parameters were CoP area $\left(\mathrm{mm}^{2}\right), \mathrm{CoP}$ velocity $(\mathrm{mm} / \mathrm{s})$. Inter-rater reliability of the equipment is $0.84-0.95$ [13].

The participants were instructed to stay on force plate with bare feet and to stand up straight while maintaining their heads towards the front side. They put their hands crossed on their chest and stood with their dominant leg while maintaining balance for 15 seconds. Data was collected by eliminating the first and last 2.5 seconds, and therefore, 10 seconds of the raw data were used. The measurements were repeated 3 times and its average was processed. The whole process took less than 5 minutes. If the subjects moved their arms to maintain balance, swayed in any side more than 10 degrees, or took their hands off their chest, opposite leg touched the ground, the measurement was performed again [14].

\section{Dynamic balance measurements}

SEBT was used to measure the subjects' dynamic balance. They put their hands on their waist while they looking straight ahead and maintained their balance with their dominant leg while they reached with their non-dominant leg in anterior, posterior-medial, posterior-lateral directions after the evaluator instructed 'go' to the subjects. Then the subjects maintained in each position for 3 seconds. The distance was measured from the center to the end of toe of the non-dominant leg by $\mathrm{cm}$. Mistakes were considered to be when the heel of the maintaining, dominant leg was off the floor, hands were taken off from their waist, or when they lost their balance. The measurement was performed 3 times for each direction and the average of the result was used as data. To get rid of the order effect, the directions were randomly given by the evaluator using Latin squares. The participants' leg length was measured from the anterior superior iliac spine to medial malleolus while they stood still. The whole process took less than 10 minutes. Inter-rater reliability of the equipment is 0.86-0.92 [15].

\section{Statistical analysis}

The data was processed by IBM SPSS Statistics ver. 21.0 software (IBM Co., Armonk, NY, USA) and mean and standard deviation values were derived from every variable. Normality test was done by Sharpiro-Wilk test and variables were analyzed using the independent t-test and paired t-test. Significant level $\alpha$ was set at 0.05 . 


\section{Results}

\section{Demographic characteristics}

The participants understood the procedure and voluntarily provided their consent. Twenty participants were randomly assigned into either the sand group $(n=10)$ or the control group $(n=10)$ and their general characteristics were as follows. There were no significant differences between the baseline characteristics of the two groups (Table 1).

\section{Static balance}

After the intervention, the sand group showed statistically significant results in every parameter ( $\mathrm{CoP}$ area, $\mathrm{CoP}$ velocity) $(p<0.05)$. However, in the control group, there was no significant result in every parameter $(p>0.05)$. There were statistically significant results in every parameter between the two groups after the intervention $(p<0.05)$ (Table 2).

\section{Dynamic balance}

There were statistically significant differences on the postero-lateral and poster-medial variables in the sand group $(p<0.05)$. In the control group, there was a statistically significant difference in the postero-lateral and anterior varia- bles $(p<0.05)$. There were no statistical differences between the two groups after the intervention $(p>0.05)$ (Table 3$)$.

\section{Discussion}

The result of this study showed the temporary effects of performing jumping lunges on a sand surface on static and dynamic balance. There were significant improvements on $\mathrm{CoP}$ area and CoP velocity in the sand group, but not in the control group. Unstable surfaces have been commonly mentioned as an important condition for balance exercises [16], and this study supports that the sand surface is an appropriate condition used for balance exercises. According to Nepocatych et al. [17], performing balance exercises on unstable surfaces improved static balance ability, which corresponds with the results of this study in that performing balance exercises on a sand surface improved static balance because the sand surface is unstable. Moreover, compared to the intervention on a firm surface, the improvement of static balance was significantly effective, and which is agreed by a study by Emery et al. [18] who compared the effect of performing balance exercises on sand and firm surfaces in healthy people.

Table 1. General characteristics of participants

$(\mathrm{N}=20)$

\begin{tabular}{lccc}
\hline \multicolumn{1}{c}{ Variable } & Sand group $(\mathrm{n}=10)$ & Control group $(\mathrm{n}=10)$ & $\mathrm{t}(p)$ \\
\hline Age $(\mathrm{y})$ & $23(3.46)$ & $22.9(2.96)$ & $0.069(0.945)$ \\
Height $(\mathrm{cm})$ & $174.8(7.3)$ & $173.3(7.76)$ & $0.445(0.661)$ \\
Weight $(\mathrm{kg})$ & $69(11.42)$ & $72.2(10.00)$ & $-0.667(0.513)$ \\
Leg length $(\mathrm{cm})$ & $88.49(4.53)$ & $89.12(3.07)$ & $-0.364(0.720)$ \\
\hline
\end{tabular}

Values are presented as mean (SD).

Table 2. Static balance variables

$(\mathrm{N}=20)$

\begin{tabular}{lccc}
\hline \multicolumn{1}{c}{ Variable } & Sand group $(\mathrm{n}=10)$ & Control group $(\mathrm{n}=10)$ & $\mathrm{t}(p)$ \\
\hline CoP area $\left(\mathrm{mm}^{2}\right)$ & & & $0.988(0.336)$ \\
Pre & $527.52(134.120)$ & $462.87(157.680)$ & $4.128(0.001)$ \\
Post & $452.497(137.055)$ & $506.38(182.227)$ & $0.391(0.701)$ \\
Changes & 75.031 & -43.508 & \\
$\mathrm{t}(p)$ & $4.421(0.002)$ & $-1.878(0.093)$ & $3.259(0.004)$ \\
CoP velocity $(\mathrm{mm} / \mathrm{s})$ & $33.721(9.876)$ & $31.87(11.266)$ & \\
Pre & $28.696(7.883)$ & -0.392 & \\
Post & 5.025 & $-0.461(0.656)$ & \\
Changes & $3.521(0.007)$ & & \\
$\mathrm{t}(p)$ & &
\end{tabular}

Values are presented as mean (SD).

CoP: center of pressure. 
Table 3. Dynamic balance variables $(\mathrm{N}=20)$

\begin{tabular}{|c|c|c|c|}
\hline Variable & Sand group $(n=10)$ & Control group $(n=10)$ & $\mathrm{t}(p)$ \\
\hline \multicolumn{4}{|l|}{ Anterior } \\
\hline Pre & $74.956(3.370)$ & $71.116(6.900)$ & $1.580(0.131)$ \\
\hline Post & $77.059(5.019)$ & $75.317(6.970)$ & \\
\hline Changes & -2.103 & -4.201 & $1.109(0.282)$ \\
\hline $\mathrm{t}(p)$ & $-1.901(0.090)$ & $-2.737(0.023)$ & \\
\hline \multicolumn{4}{|l|}{ Postero-lateral } \\
\hline Pre & $76.119(6.958)$ & $66.655(14.325)$ & $1.879(0.083)$ \\
\hline Post & 79.33 (6.914) & $70.917(16.642)$ & \\
\hline Changes & -3.211 & -4.262 & $0.578(0.570)$ \\
\hline $\mathrm{t}(p)$ & $-2.319(0.046)$ & $-3.617(0.006)$ & \\
\hline \multicolumn{4}{|l|}{ Postero-medial } \\
\hline Pre & $80.451(7.752)$ & $78.307(10.469)$ & $0.520(0.609)$ \\
\hline Post & $84.61(5.770)$ & $82.61(10.940)$ & \\
\hline Changes & -4.158 & -4.304 & $0.056(0.956)$ \\
\hline $\mathrm{t}(p)$ & $-3.339(0.009)$ & $-1.882(0.092)$ & \\
\hline
\end{tabular}

Values are presented as mean (SD).

In the usual training for improving balance ability, balance between agonist and antagonists has been highlighted $[19,20]$. Lunge exercise reinforces not only the hip and thigh muscles, but also requires co-contraction of the quadriceps and hamstring muscles [21]. It appears that the exercise in this study derived the activation of the agonist and antagonist muscles of the lower extremities, and thus, static balance ability was improved.

The gastrocnemius is activated in the support phase during a jump as one of the agonists and plays an important role in ankle strategy among the balance maintaining strategies. It seems that the decrement of sway on static balance occurred because of the activation of the gastrocnemius muscle by the repeated jumping lunge exercises.

In the sand group, there were significant improvements in the postero-lateral and postero-medial variables, and there was a significant improvement in the postero-lateral and anterior variables in the control group.

The result of improvement in direction variables before and after exercise corresponds with a previous study [22], but the results of the effect of exercise on different surfaces was not in agreement. The result of previous studies examining the effects of performing 4 weeks of balance training on dynamic balance reported that there was significant improvements in the experimental group compared to the control group [23,24]. However, it seems that the period of exercise of the study was short and the exercise was consisted of a single exercise, thus there was no difference between groups. Furthermore, there were studies that reported that exercising on sand surfaces induced more fatigue and energy expenditure after exercise than other surfaces $[25,26]$. In the study, the balance abilities were measured for one minute after the exercise, thus the participants may not have had enough time to recover from exercising on a sand surface when it was time to perform the SEBT. Moreover, the posture of the lunge was not related with SEBT. During the jumping lunge, the participants jumped straight up in the air instead of to the other directions, such as the SEBT, demanding that people need to maintain their balance in the various positions. Thus, performing jumping lunges does not improve dynamic balance ability significantly on the SEBT. Although there was no significant difference, there was a balanced improvement in both posterior directions, and therefore, balance exercises on sand surfaces appear to be more efficient. Also, exercising on a sand surface has a few advantages, such as shock absorption and less muscular soreness, which may render it to be more suitable for rehabilitation exercises, such as balance exercises for the elderly, patients with musculoskeletal problems and people who do not have enough abilities to exercise.

Jumping lunge exercises used in this study were in the low intensity category of plyometric protocols and are considered to be good for the thigh muscles, especially the quadriceps muscle. During the performance of a jumping lunge, there is a strong concentric contraction of the quadriceps muscle during the jump and is a strong eccentric contraction to maintain the weight during landing into the lunge position after switching legs in the air. These characteristics are di- 
rectly related with the dynamic balance ability for a one-legged standing position, such as a single leg squat or the SEBT [27]. During the SEBT, to maintain the body in an upright position while reaching with the other leg towards various directions against gravity requires eccentric and concentric contractions of the quadriceps muscles.

Thus, it seems that the SEBT results were significantly improved because the reaction and muscular control abilities were temporarily improved right after the jumping lunge. Also, unlike the control group, it seems that the reason why there were significant improvements in the postero-medial and lateral directions in the sand group was because the characteristic of the unstable surface made turbulence on the medial and lateral side during exercise and they caused adaptation of the quadriceps muscles to the turbulence.

However, there were a few limitations in the study as follows; 1) electromyography has not performed to prove the changes in muscular activation and 2) the number of subjects was not sufficient.

According to the results, there was a significant improvement in static balance in the sand group compared to the control group, which indicates that exercising on a sand surface is more effective time-wise. The authors recommend future researchers to include the sand surface in rehabilitation protocols to obtain an effective improvement based on the results. Furthermore, investigation of the long-term effects of the intervention needs to be proved in future studies.

\section{Acknowledgements}

This research was a part of the project titled 'The base study to discover and to commercialize for the resources of sea healing to activate marine industry', funded by the Ministry of Oceans and Fisheries, Korea.

\section{Conflict of Interest}

The authors declared no potential conflicts of interest with respect to the authorship and/or publication of this article.

\section{References}

1. Graafmans WC, Ooms ME, Hofstee HM, Bezemer PD, Bouter LM, Lips P. Falls in the elderly: a prospective study of risk factors and risk profiles. Am J Epidemiol 1996;143:1129-36.
2. Park J, Cha J, Kim H, Asakawa Y. Immediate effects of a neurodynamic sciatic nerve sliding technique on hamstring flexibility and postural balance in healthy adults. Phys Ther Rehabil Sci 2014;3:38-42.

3. Shin J, Mun MH, Chung Y. The immediate effects of patellar taping on balance and gait ability in individuals with chronic stroke. Phys Ther Rehabil Sci 2014;3:125-33.

4. Karadenizli ZI. The Effects of plyometric education trainings on balance and some psychomotor characteristics of school handball team. Univers J Educ Res 2016;4:2292-9.

5. Singh A, Sakshi G, Singh SJ. Effect of plyometric training on sand versus grass on muscle soreness and selected sport-specific performance variables in hockey players. J Hum Sport Exerc 2013;9:59-67.

6. Binnie MJ, Dawson B, Arnot MA, Pinnington H, Landers G, Peeling P. Effect of sand versus grass training surfaces during an 8 -week pre-season conditioning programme in team sport athletes. J Sports Sci 2014;32:1001-12.

7. Johnson BA, Salzberg CL, Stevenson DA. A systematic review: plyometric training programs for young children. J Strength Cond Res 2011;25:2623-33.

8. Ebben WP, Flanagan EP, Sansom JK, Petushek EJ, Jensen RL. Ground reaction forces of variations of plyometric exercises on hard surfaces, padded surfaces and in water. Int Soc Biomech Sport 2010;1:533-6.

9. Stemm JD, Jacobson BH. Comparison of land-and aquatic-based plyometric training on vertical jump performance. J Strength Cond Res 2007;21:568-71.

10. Myer GD, Ford KR, Brent JL, Hewett TE. The effects of plyometric vs. dynamic stabilization and balance training on power, balance, and landing force in female athletes. J Strength Cond Res 2006;20:345-53.

11. Gribble PA, Hertel J. Considerations for normalizing measures of the star excursion balance test. Meas Phys Educ Exerc Sci 2003;7:89-100.

12. Park J, Choi W, Lee S. Effects of immediate unilateral whole body vibration on muscle performance and balance in young adults. Phys Ther Rehabil Sci 2013;2:115-8.

13. Bauer C, Gröger I, Rupprecht R, Gassmann KG. Intrasession reliability of force platform parameters in community-dwelling older adults. Arch Phys Med Rehabil 2008;89:1977-82.

14. Hwang W, Jang JH, Huh M, Kim YJ, Kim SW, Hong IU, et al. The effect of hip abductor fatigue on static balance and gait parameters. Phys Ther Rehabil Sci 2016;5:34-9.

15. Gribble PA, Kelly SE, Refshauge KM, Hiller CE. Interrater reliability of the star excursion balance test. J Athl Train 2013;48: 621-6.

16. Horak FB, Wrisley DM, Frank J. The balance evaluation systems test (BESTest) to differentiate balance deficits. Phys Ther 2009; 89:484-98.

17. Nepocatych S, Ketcham CJ, Vallabhajosula S, Balilionis G. The effects of unstable surface balance training on postural sway, stability, functional ability and flexibility in women. J Sports Med Phys Fitness 2018;58:27-34.

18. Emery CA, Cassidy JD, Klassen TP, Rosychuk RJ, Rowe BH. Effectiveness of a home-based balance-training program in reducing sports-related injuries among healthy adolescents: a cluster randomized controlled trial. CMAJ 2005;172:749-54. 
19. Kollmitzer J, Ebenbichler GR, Sabo A, Kerschan K, Bochdansky T. Effects of back extensor strength training versus balance training on postural control. Med Sci Sports Exerc 2000;32:1770-6.

20. Kaji A, Sasagawa S, Kubo T, Kanehisa H. Transient effect of core stability exercises on postural sway during quiet standing. $\mathrm{J}$ Strength Cond Res 2010;24:382-8.

21. Hefzy MS, al Khazim M, Harrison L. Co-activation of the hamstrings and quadriceps during the lunge exercise. Biomed Sci Instrum 1997;33:360-5.

22. Paterno MV, Myer GD, Ford KR, Hewett TE. Neuromuscular training improves single-limb stability in young female athletes. J Orthop Sports Phys Ther 2004;34:305-16.

23. Eisen TC, Danoff JV, Leone JE, Miller TA. The effects of multiaxial and uniaxial unstable surface balance training in college athletes. J Strength Cond Res 2010;24:1740-5.

24. Hale SA, Fergus A, Axmacher R, Kiser K. Bilateral improvements in lower extremity function after unilateral balance training in individuals with chronic ankle instability. J Athl Train 2014;49:181-91.

25. Binnie MJ, Dawson B, Pinnington H, Landers G, Peeling P. Effect of training surface on acute physiological responses after interval training. J Strength Cond Res 2013;27:1047-56.

26. Muramatsu S, Fukudome A, Miyama M, Arimoto M, Kijima A. Energy expenditure in maximal jumps on sand. J Physiol Anthropol 2006;25:59-61.

27. Earl JE, Hertel J. Lower-extremity muscle activation during the Star Excursion Balance Tests. J Sport Rehabil 2001;10:93-104. 